\title{
Las agrupaciones vecinales en defensa de los barrios. La construcción política desde lo local
}

\author{
Eduardo Canteros Gormaz
}

Universidad Alberto Hurtado, Santiago, Chile. Email: ecantero@uahurtado.cl

Resumen: La construcción masiva de edificios en algunas comunas de Santiago, la construcción de autopistas o el ensanche de avenidas son algunas de las intervenciones públicas y/o privadas que se han realizado en algunos barrios y que han despertado el malestar de sus vecinos. Estos se han organizado en agrupaciones vecinales las cuales han adquirido relevancia en la discusión local sobre desarrollo urbano en los últimos años. Este artículo propone una línea conceptual para leer a dichos grupos desde la heterogeneidad de su discurso, intentando con esto comprender su acción colectiva y su proyección en la agenda urbana.

Palabras clave: barrio, agrupación de vecinos, agenda urbana.

\section{The neighbor groups in defence of their neighborhoods: The building of politics from local scale}

\begin{abstract}
The massive construction of buildings in some communes of Santiago, the construction of haighways or the widening of streets are some public and/or private interventions that have aroused the uneasiness of their neighbors. They have organized themselves into neighborhood groups which have gained prominence in e local discussion on urban development in recent years. This article proposes a conceptual line to read these groups from the heterogeneity of their discourse, for understanding their collective action and their effects on the urban agenda.
\end{abstract}

Key words: neighborhood, neighborhood group, urban agenda.

\section{As organizações de bairro em defesa dos municípios. A construção da política local.}

Resumo: A construção maciça de edifícios em alguns bairros de Santiago, a construção de estradas ou alargamento de ruas são algumas das intervenções públicas e / ou privadas que tenham sido feitos em alguns bairros e que têm despertado a inquietação dos seus vizinhos. Estes foram organizados em grupos de bairro que têm ganhado destaque no debate local sobre o desenvolvimento urbano nos últimos anos. Este artigo propõe uma linha conceitual para ler os grupos da heterogeneidade de seu discurso, tentou compreender esta ação coletiva e seus efeitos sobre a agenda urbana.

Palavras-chave: bairro, grupo de vizinhança, agenda urbana. 


\section{Santiago en disputa}

Santiago es un territorio en disputa local. Así lo evidencia una serie de conflictos que a lo largo y ancho de la ciudad relevan a algunos Barrios, donde vecinos por un lado y autoridades e inmobiliarias por otro, animan un conflicto sobre el destino de dichos territorios.

Estos conflictos son una manifestación clara que la ciudad es un lugar político, donde diferentes proyectos chocan, se implementan y discuten construyendo diferentes campos en disputa en la ciudad. En el caso de Santiago, discusiones sobre infraestructura, servicios, la existencia de un alcalde mayor o su extensión, crean distintos campos de conflicto, los cuales legitiman o no a diferentes actores para participar en las conversaciones.

Para este artículo nos referiremos a la disputa que se da en torno al Barrio. Dicho concepto o escala geográfica se puesto en el centro de debate desde mediados de los años noventa con la organización ciudadana que se opuso al trazado original de la Costanera Norte en su paso por los Barrios de Bellavista, Pedro de Valdivia Norte y la Vega. En la actualidad, dicha organización vecinal se ha constituido en una ONG preocupa+da de temas urbanos, y los conflictos locales se despliegan en diferentes Barrios de comunas como Ñuñoa, Providencia, Vitacura, La Reina, San Miguel, La Florida, Santiago Centro y Estación Central.

Estos sectores de Santiago han visto emerger agrupaciones o comités de vecinos que buscan "defender" el Barrio de las intervenciones que, a su juicio, destruyen la trama urbana y comunitaria de dichos territorios. Este levantamiento pone de manifiesto la construcción de una ciudadanía activa en la defensa de sus derechos territoriales, y por otro, releva una escala (el Barrio) como una entelequia con atributos positivos.

Frente a esto surgen las preguntas de si cada barrio es un proyecto independiente dentro de la ciudad común y qué factor determinaría que tal o cual barrio deba primar sobre el resto, difundiendo su idiosincrasia como modelo a otros territorios de la ciudad. En otras palabras, en estas disputas nos enfrentamos a la paradoja de la defensa de lo particular como atributo general, y este artículo espera mostrar una matriz de análisis que permita aclarar cómo afrontar esta paradoja, avanzando en la comprensión de estas agrupaciones de vecinos.

\section{Los vecinos en la defensa de sus barrios}

Conflictos como los descritos aparecen por todo Santiago, y según algunos antecedentes entregados por investigadores, centros de estudios y medios de comunicación existen cerca de 100 puntos de conflicto en la ciudad de Santiago, entre los que se cuentan los asociados al crecimiento urbano, al uso y apropiación de espacios urbanos, al medio ambiente, a la vivienda y a daños del terremoto. ${ }^{1}$ 
Según Poduje (2008), si bien estos conflictos se concentran principalmente en los sectores de altos ingresos socioeconómicos, en los últimos años dicha tendencia ha cambiado, naciendo otras organizaciones vecinales en sectores de ingresos socioeconómicos medios y bajos. En su conjunto, estos conflictos, sin contar las multas y los atrasos que han generado a los proyectos y dejando de lado aquellos proyectos no realizados, han generado cambios por un costo total aproximado de US\$ 586 millones (Poduje, 2008).

Pese a su heterogeneidad temática, una de las constantes de estos conflictos es la modalidad de Participación Ciudadana establecida para la elaboración y modificación de los Planes Reguladores Comunales.

La principal debilidad de los Planes Reguladores comunales en materia de Participación Ciudadana está en el nivel de involucramiento de los vecinos. Si bien se establece en el artículo 43 de la Ley General de Urbanismo y Construcción que para el desarrollo de un Plan Regulador Comunal se requerirá de la participación de los vecinos, esta no llega más allá de la consulta, no involucrando a los vecinos en procesos de diseño participativo de su territorio (Mlynarz, 2007). Esta situación tiene una evaluación diferenciada para cada uno de los actores de este proceso, y mientras las autoridades consideran la Participación como un trámite a realizar, las organizaciones vecinales tienen la expectativa de poder avanzar en profundizar su participación (Mlynarz, 2007).

En la población La Victoria, según cuenta en una entrevista una dirigenta, durante el año 2007 se enteraron de que se realizaría una modificación al Plano Regulador Comunal donde se encancharían avenidas y permitiría la construcción de edificios de cinco pisos. Frente a esto la comunidad se organizó, y con el apoyo técnico de una ONG, desarrollaron una serie de talleres los cuales terminaron con una propuesta de desarrollo con criterios vecinales y que permitía la solución a su principal problema: la vivienda para los allegados. El resultado final fue que la modificación al Plan Regulador se detuvo, y no prospero ni la ideada desde el municipio, ni la generada desde los vecinos.

Por otro lado, vecinos de la calle Viel con Av. Matta vieron durante el 2008 que se anunciaba la construcción de un edificio de 22 pisos a pasos de sus barrios. Revisaron el Plan Regulador con detalle, y vieron - a su juicio en medio de la letra letra chica - una modificación efectuada el año 2003 donde se permitía, cumpliendo ciertas condiciones técnicas, que se construyeran edificios de esa altura en el barrio. Según cuenta un entrevistado, partían de la base de considerar valido por parte de los vecinos vender sus viviendas, así como de las empresas desarrollar sus proyectos, pero que sin embargo esto debería hacerse respetando al resto de los vecinos y pagando un precio justo por la vivienda. Por esta razón se propusieron ser declarados zona patrimonial y así dar valor homogéneo a su barrio. Finalmente el día 5 de Junio de 2009 se dicta el Decreto que lo designa zona patrimonial. 
Esta oposición por parte de los vecinos a los cambios que ocurren en su barrio puede entenderse de diferentes maneras: reacción a las molestias transitorias provocadas por los nuevos cambios; reacción frente a la pérdida de valor de sus propiedades; desagrado frente a la llegada de nuevos vecinos; rechazo de los cambios paisajísticos del barrio. Estas y otras razones pueden estar a la base del rechazo de los vecinos al cambio, pero lo que es interesante destacar es que muchos de estos grupos han pasado del rechazo a la propuesta, es decir, de la oposición a los cambios han transitado hacia la elaboración detallada de planes de desarrollo para sus barrios y comunas.

En síntesis, a lo largo y ancho de la ciudad grupos de vecinos organizados informalmente han entrado en la discusión sobre el desarrollo y planificación urbana de sus territorios, y lo han hecho vinculando protesta y propuestas, a partir de las cuales se dibuja el barrio que desean y la ciudad en la cual quieren y esperan vivir.

\section{Construcción de la disputa urbana}

La discusión pública sobre el destino urbano del Barrio Yungay, sobre la Población La Victoria o sobre Vitacura no nació únicamente de un proyecto municipal o de un diagnóstico sectorial sobre alguna necesidad urbana, sino más bien nació de la reacción organizada de un grupo de vecinos. Así, el escenario de la discusión pública sobre el destino de muchos barrios de Santiago no se constituye por el despliegue territorial de las iniciativas públicas o privadas ${ }^{2}$, sino más bien con los procesos de contestación que a lo largo y ancho de Santiago desarrollan múltiples grupos de vecinos.

Así, un tema dominado tradicionalmente desde la esfera técnica comienza a ser cuestionado por un nuevo actor, tradicionalmente ausente de la mesa de conversación. De este modo, se despliega una controversia, donde el carácter técnico de la planificación urbana se pone en cuestión, nuevos actores entran a la discusión no sólo demandando más información sino que también generando nuevos criterios. Finalmente, la planificación y gestión del territorio sale de los gabinetes técnicos y entra al plano de la discusión política ${ }^{3}$.

El resultado es que la diada servicio público - inversión privada no es la que entrega el resultado óptimo para la ciudad, sino que son necesarias las especificidades locales encarnadas en sus vecinos las que tienen que entrar en la conversación para asegurar la representación política y material de la heterogeneidad urbana.

Hay que destacar además que esta emergencia de las agrupaciones vecinales como un actor en la discusión sobre la Planificación Urbana Local, se da en un contexto de cambios en la acción colectiva de la ciudad. Así, si tradicionalmente las marchas y las tomas eran las principales modalidades de protesta de los actores sociales urbanos y su vinculación con los 
partidos políticos era el mecanismo que encontraban para pasar desde sus necesidades particulares a la exigencia de un derecho general (Espinoza, 1998), ahora los partidos políticos pierden relevancia como vehículo de demandas y la heterogeneidad socioeconómica de sus sus participantes y acciones es una de sus principales características (Sabatini y Wormad, 2004)

Por un lado la organización contestataría y propositiva de las agrupaciones vecinales y por otro el cambio de escenario de la acción colectiva en la ciudad, nos obliga a reflexionar sobre la nueva ciudadanía que esta formación, cómo entenderla y cómo proyectar su alcance. Por ejemplo es posible entender todas estas protestas vecinales como un tipo de actor urbano, o estamos frente a una serie de organizaciones emergentes e individuales.

En busca de avanzar en sus respuestas, analizaremos en primer lugar la construcción que estas agrupaciones realizan de los problemas públicos para luego reconocer los proyectos políticos que emergen de alguna de estas agrupaciones vecinales.

\section{Las nuevas arenas públicas y los proyectos políticos desde el vecindario}

Tal como hemos planteado anteriormente, los grupos de defensa del barrio se originan desde la protesta y oposición a intervenciones a sus territorios (construcción masiva de edificios en altura, el ensanche de una avenida o la instalación de una autopista). A partir de este evento, el grupo se organiza y despliega una serie de actividades de protesta y propuesta.

Si tomamos la secuencia que va desde la molestia específica de cada vecino, lo que generan conversaciones entre conocidos o amigos, hasta llegar a la manifestación pública y organizada del malestar podemos ver un elemento de central interés, cual es la conversión de un problema particular/personal en un tema público, que le compete a toda la comunidad, y por consiguiente a las autoridades pertinentes.

El resquebramiento de la pared de una casa donde habita una mujer mayor de edad por efecto de la construcción contigua de los cimientos de un edificio en altura se convierte en el punto de origen de una protesta comuna contra la masacre urbana que se realiza en la comuna al permitir la construcción masiva de edificios en altura en una comuna tradicionalmente poblada de casas unifamiliares. De la pared agrietada a la protesta masiva por la modificación del Plano Regulador Comunal.

Este transito de lo privado a lo público, así como la definición de ambos polos viene a constituir las nuevas arenas públicas (Cefaï, 2005), donde el elemento destacado pasa justamente en la manera como los grupos activamente definen qué problema privado se convierte en un tema 
público, llamando la atención de las autoridades y legisladores (Lichterman y Cefaï, 2005).

El proceso de traducción entre lo privado y lo público da cuenta del uso por parte, en este caso, de las agrupaciones de vecinos de diferentes lógicas de justificación y de la constitución de bienes morales que obliga a evaluar diferentes alternativas económicas para garantizar el bien común, el cual es expresado por estos grupos a través de la movilización del malestar popular (Cefaï, 2005).

El sacar áreas verdes, el cambio de una plaza abierta por un gimnasio privado, las estrategias de las inmobiliarias para lograr que los vecinos vendan sus casas, las reuniones en las esquinas de los vecinos indignados, la elaboración de las pancartas. Toda esa secuencia genera un argumento que justifica, a la luz de los participantes, la lucha en contra del problema público creado en su vecindario. Pero no sólo eso, es un argumento de tal validez, que tiene el poder de mandato para otros vecinos de apoyar su lucha y obliga a la autoridad comunal a responder.

Así, el argumento creado por la agrupación vecinal orienta las acciones del grupo, pero también pone sobre la mesa barrial, valores e historia, que le da un sustrato cultural a la lucha emprendida.

Para Dagnino, Olvera y Panfichi el proyecto político “[nos muestra] el vínculo indisoluble entre la cultura y la política, [no reduciéndose] a estrategias de actuación política en el sentido estricto, sino que expresan, vehiculan y producen significados que integran matrices culturales más amplias” (2006: 44).

El proyecto "no se limita a formulaciones sistematizadas y abarcadoras como los proyectos partidarios, por ejemplo, sino que recubre una amplia gama de formatos en los cuales las representaciones, creencias e intereses se expresan en acciones políticas con distintos grados de explicitación y coherencia” (Dagnino, Olvera y Panfichi, 2006:45)

De esto se desprende que el énfasis no estaría en la identificación clara e inequivoca de actores políticos con planes y estrategias para el logro del poder, sino más bien en la construcción de un mapa de significados que encarnan proyectos políticos, los cuales no siempre son explícitos ni tampoco se expresan coherentemente.

Pese a que los significados culturales que emergen de muchas de estas organizaciones se vincula con intentar quebrar la hegemonía cultural del neoliberalismo (Lichterman y Cefaï, 2005), hay que rescatar los significados múltiples y heterogéneos que levantan los grupos vecinales, resguardándonos así de no cometer el mismo error o ilusión que se cometió con la conceptualización de los Nuevos Movimientos Sociales en los años 70, donde lo que primaba era el esfuerzo analítico por mostrar la unidad de intereses, y con esto apoyar proyectos políticos partidistas (Cefaï, 2005). 
Dagnino, Olvera y Panfichi dan cuenta de al menos tres proyectos políticos en las sociedades latinoamericanas: el autoritario, el democrático participativo y el neoliberal. Analicemos cada uno de ellos para armar una estructura clara de los aspectos que evidencian.

El proyecto autoritario no sólo nos habla de la presencia de una figura dictatorial al mando del poder, sino por sobre todo de "una cultura que legitima las diferencias sociales, que internalizan los códigos que jerarquizan a las clases y los grupos sociales y los organizan en categorías con base en su pertenencia de clase, raza, género, región y país.”(Dagnino, Olvera y Panfichi, 2006:50).

En otras palabras, la naturalización de la diferencias sociales está a la base de este proyecto, lo que tiene implicancias en el rol y funciones del Estado, especialmente de aquellos instrumentos que busquen terminar o disminuir aquellas diferencias sociales.

Por su parte proyecto democrático - participativo, "su núcleo esencial está conformado por una concepción de profundización y radicalización de la democracia, que confrontan con nitidez los límites atribuidos a la democracia liberal representativa como sistema privilegiado de las relaciones entre Estado y sociedad. Así, para hacer frente al carácter excluyente y elitista de este paradigma se defienden los modelos de democracia participativa y deliberativa como complementarios a aquél [donde la participación] ayudaría a una desprivatización del Estado [concibiéndose] fundamentalmente, como compartir el poder de decisión del Estado sobre los asuntos relativos al interés público. Por lo tanto, se distingue de una noción de participación que se limita a la consulta de la población” (Dagnino, Olvera y Panfichi, 2006: 54).

La Participación sería entonces para este modelo la clave de relación entre la sociedad civil y el Estado, lo que implica el esfuerzo por el establecimiento de mecanismos de diálogos claros y vinculantes entre ambas partes. Para Espinoza (2004) la clave estaría dada por el establecimiento claro y explícitamente negociado de una interfaz entre las partes, que de cuenta del cambio de contrato con la ciudadanía, cada vez más constituida por redes comunitarias horizontales, diversas y de baja jerarquía, que buscan coordinación más que negociación o conflicto.

Finalmente el proyecto neo liberal, parte de la base que "la primacía del mercado en tanto eje reorganizador de la economía es vista como algo que debe extenderse al conjunto de la sociedad. La búsqueda de eficiencia y de modernización pasa entonces a legitimar la adopción del mercado como organizador de la vida social y política. La transferencia de la lógica del mercado al ámbito estatal transforma a los gobiernos en "proveedores de servicios” y a los ciudadanos en "clientes" o "usuarios”, en relación con los cuales es preciso tener sensibilidad respecto a sus demandas y eficiencia en la atención” (Dagnino, Olvera y Panfichi, 2006: 61). 
Para los autores, este proyecto tiene a la base una minimización de la política que no reconoce a una ciudadanía constituida a partir de derechos universales, sino que como atributo de esfuerzos emergentes y focalizados de organizaciones específicas de ciudadanos. La contracara de este proceso, es decir el Estado como garante de derechos ciudadano, es reducido en su rol, y es el mercado quien distribuye los recursos entre las organizaciones emergentes.

Así, se pasa de la sociedad civil, al concepto de tercer sector “al lado del mercado y del Estado, indica la nueva función y designa el intento de retirar de la sociedad civil su papel como constitutiva del terreno de la política, ahora otra vez condensado en la sociedad política. Autodeterminado, como apolítico, el tercer sector refuerza una concepción estatista del poder y de la política, precisamente contra la cual se dirigió la visión de sociedad civil del proyecto democrático, al confrontar al monopolio del Estado y de la sociedad política en el ejercicio de la política y del poder” (Dagnino, Olvera y Panfichi, 2006: 64).

Finalmente, y luego de revisar los planteamientos conceptuales sobre los proyectos políticos, podemos plantear que éstos transmiten matrices culturales más amplias y que para el caso de Latinoamérica movilizan los siguientes significados: origen y legitimidad de las diferencias sociales; estilos y mecanismos de relación entre ciudadanía y el Estado; y finamente la ciudadanía como un atributo general o como beneficio al mérito individual/organizacional emergente.

\section{El problema público en los barrios}

La defensa del barrio es el enunciado bajo el cual se organizan las agrupaciones vecinales que hemos citado en este artículo, y donde aparentemente se vería homogeneidad e igualación de objetivos, es posible desentrañar heterogeneidad, y es que pese a compartir el nombre o la intensión general, cada barrio, cada sector de la ciudad y cada organización hace alusión a su historia, a su posición dentro de la ciudad, a su contexto socioeconómico.

Así el enunciado está lleno de otras voces - ajenas como diría Bajtin - las cuales son ordenadas, priorizadas y desplegadas en un argumento por cada organización, el cual luego es hechas públicas y confrotado para organizar e ir construyendo su lucha. ${ }^{4}$

En el caso de Ñuñoa y Vitacura, frente a la construcción en el primer caso, y la posible autorización, en el segundo, de construcción masiva de edificios, el problema público fue nombrado como Masacre Urbana o Aberración Urbanística respectivamente, y se refiere a que por la llegada de los edificios en altura se rompía la identidad urbanística de un sector. Ahí donde existían casas unifamiliares, llegarán edificios en altura que interrumpirían el estilo y la composición del barrio. 
A esto, el caso de Ñuñoa agrega que la llegada de los edificios de altura generará la expulsión de los vecinos con menos recursos, tercera edad y familias jóvenes hacia otras comunas rompiendo así, la heterogeneidad socioeconómica y etaria que tendría la comuna.

Este problema nace o se vincula en su desarrollo con dos acciones muy criticadas por parte de los vecinos. La primera es la conducta seguida por algunas inmobiliarias que presionan a los vecinos para que vendan sus casas, incluso sostienen, en base a mentiras. O, en el caso de Vitacura, donde los vecinos beneficiados con los cambios a la regulación serian dueños de más de algún sitio, convirtiéndose en una suerte de especuladores, quienes obtendrían grandes beneficios a costa de perjudicar al resto.

Así tanto la masacre urbana como la aberración urbanística se vincula principalmente con la destrucción de un estilo urbano de la comuna, y la consiguiente desintegración social que esto genera.

En el caso del Barrio Yungay, el problema no se vincula con la llegada masiva de edificios ${ }^{5}$ en altura, sino más bien con el régimen de extracción de la basura del barrio, donde el alcalde tomo la decisión inconsulta a la comunidad de cambio de dicho régimen, pasando de un retiro diario a un retiro tres veces a la semana.

La agrupación de defensa del Barrio Yungay construye así el problema de la basura como piedra inicial, el cual no sólo sería un problema de sanidad pública, sino que también, un problema de distancia entre el municipio y los vecinos, y sobre este último siempre es posible sospechar que nacerán otros problemas. La distancia entre la autoridad y los ciudadanos es visto así como la fuente de problemas de la democracia y como perdida de legitimidad del alcalde y concejo.

Los tres casos muestran al municipio como un actor generador o amplificador del problema, en tanto con sus acciones perjudicaría a la población de una parte de su comuna o promovería el desarrollo de proyectos que benefician sólo a pocos en desmedro de la mayor parte de la población de los sectores afectados.

La presencia del Municipio como generador o amplificador del problema lo ubica al medio del conflicto, en tanto para los vecinos, su responsabilidad pública es la defensa de todos los habitantes - o al menos la mayoría de ellos - y lo que estaría realizando iría en la línea contraria, a saber, beneficiando a unos pocos o realizando un mal negocio para el municipio.

En relación al concepto de Barrio, en las tres declaraciones públicas aparece con un doble carácter - incluso contradictorio - en tanto para Ñuñoa como para Yungay, el Barrio es el contexto espacial donde ocurren los problemas así como un objetivo a construir. Los problemas de retiro de la basura así como la llegada de edificios en altura ocurre en el barrio, por su parte la participación ciudadana ocurre y ocurrirá en el barrio, pero ade- 
más, gracias a dicha participación el barrio se construye. Este no es sólo una escala, sino que un espacio a construir con la participación de los vecinos.

Coherente con esto son las actividades que estos grupos realizan para promover el apoyo a su causa, la cual muchas veces conlleva la participación de sus vecinos, pero también la llegada de vecinos de otros sectores de Santiago. En este contexto, las actividades artísticas (música, canto y teatro) como el Carnaval de Todos los Barrios efectuado en Ñuñoa el pasado 20 de Noviembre, así como la fiesta del Roto Chileno organizado todos los años en el barrio Yungay en el mes de enero.

Estas fiestas, con la participación masiva de vecinos de diferentes partes de Santiago recrea el significado de cada barrio, pero también muestra el barrio como un significado extra espacial, donde contenidos como la solidaridad y su trabajo se constituirían como una alternativa para la ciudad homogénea.

Por su parte, en el caso de Vitacura, el barrio aparece con un significado espacial más acotado, vinculado al hogar y la familia. Así, corromper el barrio con la llegada de edificios es vincular el problema con cada uno de los hogares, y con esto con los valores que las familias buscaron al irse a vivir a una comuna con estilo unifamiliar.

\section{Los barrios dentro del barrio}

Si bien, los grupos analizados son parte de una oleada de conflictos en la ciudad de Santiago, es posible afirmar que dichos grupos tienen puntos de encuentro, así como distancias entre sus postulados y demandas.

La denuncia de los barrios plantea algunas veces de qué manera dicho territorio es perjudicado, o cómo algún sector de este es postergado para beneficiar a otro. Así, el Barrio aparece como una manera de entender las diferencias socioeconómicas y/o demograficas.

De los ejemplos que aquí exponemos, Guillermo Franke en Ñuñoa es quien da más evidencia al respecto, ya que en su declaración pública plantean que la llegada de edificios en altura obligaría a la población más pobre así como a los adultos mayores a emigrar de la comuna.

Pero además el Barrio aparece como mix social ideal, donde los servicios podrán abastecer al necesidad de todo tipo de población. Así el Barrio aparece como el retorno a esa comunidad perdida dentro de la ciudad presentes en la literatura de sociología urbana de fines del siglo XIX (Tönnies).

Por su parte, el Barrio Yungay se refiere a la diferencia internas al plantear que la autoridad comunal trataría de manera desigual a diferentes 
sectores dentro del barrio, donde la variable organización comunitaria sería el criterio que marcaría la diferencia. Por tal razón, ahí donde la comunidad se organice y presione tendrá más apoyo de parte de la alcaldía.

Así, el municipio aparece siempre propuesto como una institución ideal, que debería estar presente para asegurarle servicios de calidad a toda la población sin diferencias, así como para asegurar un trato igualitario a todos los sectores del barrio. Frente a esta imagen, el Municipio siempre parte con un déficit para estas agrupaciones.

Por otro lado, el barrio aparece también como el espacio desde el cual se discute la relación entre los vecinos, el municipio y el Estado.

Tomando el ejemplo de Ñuñoa, el instrumento del Plano Regulador Comunal es el marco de fondo cuestionado por los vecinos en tanto promovería la llegada irrestricta de capitales inmobiliarios. A esto se suma que el instrumento no cuenta con Participación Ciudadana. De tal manera que la participación informada de los vecinos permitiría controlar al Municipio y a las inmobiliarias, entregando criterios que compitan o ponderen a la rentabilidad económica al momento de decidir cómo crecerá la comuna.

Por su parte el Barrio Yungay, también pone en la participación un eje central en el análisis del problema que los aqueja, pero también como parte de la solución. La capacidad de movilización de los vecinos fue la que puso el problema en la agenda pública, pero además la participación permitirá informar a la comunidad y evaluar el desempeño edilicio en una consulta pública.

Pero más allá de eso, la participación de los vecinos se vuelve central como parte de la solución del problema. Esto se logra mediante una instauración de los vecinos como agentes técnicos relevantes, y son ellos los que ofrecen superar el problema con una propuesta técnica, en el caso del Barrio Yungay, para el desarrollo de un plan de reciclaje en el Barrio.

Este status técnico de los vecinos, también aparece en la agrupación de Vitacura, quienes no sólo discuten que la autoridad municipal no les permitió participar en sus decisiones, sino que le enrostran que no les haya brindado el apoyo técnico necesario para que pudieran presentar una propuesta alternativa a la presentada por el Municipio. Así en Vitacura no se habla explícitamente de abuso o de la llegada de agentes externos que expulsarán a los vecinos antiguos, sino la falta de apoyo municipal para que los vecinos organizados ejerzan su rol de control y de promotor del desarrollo de su comuna.

Para la consolidación de este carácter técnico de la organización de vecinos, estos acuden a la experiencia de sus dirigente y sus colaboradores más cercanos (cabe destacar que en la mayoría de los casos, los dirigentes vecinales son profesionales universitarios). Y que dentro de sus colaboradores se puede contar una red de organizaciones o coordinadora de grupos, 
los cuales además de servir de aprendizaje colectivo, permiten tener acceso a conocimiento técnico en diferentes materias.

Así, la llegada a la mesa de discusión de este actor, es la mayoría de los casos, sinónimo de sentar a la mesa a una red de organizaciones que apoyarán frente a alguna movilización, pero que por sobre todo entrega conocimiento sobre situaciones similares (en y fuera de Santiago), sobre cómo analizar el problema y también con alternativas de solución.

\section{Conclusiones}

Lo que aquí se entrega es parte de un trabajo mayor realizado en el marco de una tesis doctoral, y tiene por objetivo mostrar algunas de las categorías de análisis que sean de utilidad para analizar la paradoja en la que esta inserta la defensa de los barrios.

Así, la defensa de un territorio particular como atributo general para la ciudad determina la heterogeneidad de sus demandas y objetivos. La defensa basada en la experiencia particular de cada uno de los barrios, repercute que los valores generales a los que cada grupo apela varía a la luz de la historia, ubicación y contexto de cada barrio.

No estamos diciendo con esto que cada agrupación vecinal sea una empresa particular, sino más bien que la riqueza de su arribo como actor urbano destacado, se basa en la heterogeneidad interna. La cual le permite llegar a una mayor cantidad de lugares de la ciudad, pero que también muestra en parte su debilidad.

Lo que hemos intentado mostrar es que en algunos casos el problema público es construido como una ruptura de un estilo, como la perdida de homogeneidad en las construcciones, mientras que desde otro barrio, el problema está en la salud pública y en la distancia entre ciudadanía y autoridades comunales.

La diversidad también es posible observarla en el tratamiento que cada uno de los grupos hace de las diferencias internas de su barrio. Mientras para algunos lo que hay que defender es la heterogeneidad interna, para otros, el valor esta puesto en la homogeneidad de sus habitantes.

Es quizás el rol de la ciudadanía organizada es el elemento más rico en significados entre las agrupaciones de vecinos. Todas lo relevan, no sólo como forma de evidenciar los problemas, sino como parte de la solución. Sin embargo para el Barrio Yungay la participación llevará a construir a un actor político que controle y converse con el municipio de igual a igual, y por su parte para Vitacura, el municipio debería prestar todo el apoyo necesario para que la comunidad proponga lo que debe ocurrir en sus territorios. 
El Barrio finalmente aparece con grandes encuentros y desencuentros. Para todos el barrio es una suerte de comunidad perdida dentro de un Santiago cada vez más global, sin embargo tanto para Ñuñoa como para Yungay el barrio no es solo un contexto donde se despliegan los problemas y sus acciones, sino que es un objetivo por construir, y la participación de la comunidad no es sólo para marcar los problemas que el municipio debe resolver, sino para construir un nuevo y mejor barrio.

En cambio, para Vitacura, la vinculación del barrio con el hogar lo deja más circunscrito a un tema privado y doméstico, donde prima la igualdad familiar, y no la heterogeneidad social y política.

Sintetizando, el enunciado defensa del barrio muestra un nuevo actor en la discusión sobre la planificación urbana local, el cual se ha empoderado no sólo adquiriendo información, sino que como generador de criterios para el desarrollo local. Pero también, dicho enunciado es una suerte de rompecabezas, donde cada barrio - desde su historia, ubicación y condición - pone su voz, como un efecto refractario de sus habitantes.

Así, el análisis de la acción colectiva en la ciudad debe considerar ambos registros, y construir desde la diversidad, y hasta contradicción, la historia ciudadana de Santiago del bicentenario. 
Polis, Revista de la Universidad Bolivariana, Volumen 10, $N^{\circ} 28,2011$

\section{Notas}

${ }^{1}$ Según trabajo desarrollado por la fundación de Estudios Sur en su área de Ciudad, Barrio y Organización, y que se puede visitar en la página: http://mapadeconflictos.sitiosur.cl/ index1.php

${ }^{2}$ Es interesante destacar que esta discusión se da de manera paralela a la implementación de un programa gubernamental llamado "Quiero mi Barrio” donde sí es posible observar una discusión participativa sobre el destino de los barrios, sin embargo, los ejemplos que aquí se trabajan no emergen de este programa barrial, sino que de la propia organización de los vecinos frente a una amenaza externa.

${ }^{3}$ Sobre las controversias socio técnicas y su capacidad de reconstruir la realidad social, se sugiere revisar los aportes de Michell Callon y Bruno Latour.

${ }^{4}$ A continuación se ejemplificará con tres agrupaciones vecinales, específicamente, con la declaración pública inicial que aparece en su página web o blog. En primer lugar la Agrupación Barrial Guillermo Franke y su declaración No a la Masacre en Ñuñoa del 2006 disponible en la página http://guillermofranke.blogspot.com/2006_05_01_archive.html; luego la agrupación por la Defensa de Vitacura, en especial su declaración Rebelión Vecinal del 1 de Septiembre de 2006 disponible en la página http://salvemosvitacura.blogspot.com/; y finalmente la organización Vecinos por la defensa del Barrio Yungay y su Manifiesto por la Defensa del Barrio Yungay del 1 de Agosto de 2006, disponible en la página http:// www.elsitiodeyungay.cl/index.php?option=com_frontpage\&Itemid=1\&limit= 27\&limitstart=1161\&lang $=$.

${ }^{5}$ Esto no significa que la llegada masiva de edificios en altura que rompen con la armonía de estilo y la composición socioeconómica del Barrio no exista como problema, es parte del problema que ha construido la organización a lo largo del tiempo. Lo que se aclara en este caso es el problema inicial a partir del cual se organiza el Blog. 


\section{Bibliografía}

Cefaï, D. (2005), “Os novos movimentos de protesto en Franca. A articulacao de novas arenas públicas”, Revista Crítica de Ciencias Sociales, 72. Octubre: 129 - 160.

Dagnino, E., Olvera, A. \& Panfichi, A. (2006), La disputa por la construcción democrática en America Latina. Fondo de Cultura Económica, Centro de Investigaciones y Estudios Superiores en Antropología Social, Universidad Veracruzana.

Espinoza, V. (2004), De la política social a la participación en un nuevo contrato de ciudadanía, en revista Política, Santiago de Chile / Vol.43 Primavera. 149-183.

Espinoza, Vicente (1998), "Historial social de la acción colectiva urbana: Los pobladores de Santiago, 1957 - 1987”, EURE, V. 24, № 72, Santiago, Chile.

Lichterman, P \& Cefaï, D. (2005), “The idea of Political Culture”, en The Oxford Handbook of work of Contextual Political Analysis, ed. R.Goodin \& Ch. Tilly. Oxford University Press.

Mlynarz, D. (2007), “Participación Ciudadana en el proceso de Diseño y Modificación de los Planes Reguladores Comunales. Propuesta desde diversos actores”, Tesis Magister en Política y Gobierno. Universidad de Concepción - FLACSO / Chile.

Poduje, I. (2008), "Participación ciudadana en proyectos de infraestructura y planes reguladores”, Temas de la Agenda Pública, Vicerrectoría de Comunicaciones y Asuntos Públicos PUC, Año 3,No. 22.

Sabatini, F. \& Worlmald, G. (2004), "La Guerra de la basura de Santiago: desde el derecho a la vivienda al derecho a la ciudad”, EURE, V.30, No 91, pp. 67 - 86. Santiago

Recibido: 24.01.2011

Aceptado: 03.03.2011 